\title{
Eccentricity Estimation in Ultra-precision Rotating Devices based on a Neuro-fuzzy model
}

\author{
Raúl M. del Toro ${ }^{1}$, Rodolfo E. Haber ${ }^{2}$ \\ ${ }^{1}$ ETSIS de Telecomunicación \\ Universidad Politécnica de Madrid. \\ Campus Sur UPM km. 7. E-28031. Madrid. Spain \\ raul.deltoro@upm.es \\ ${ }^{2}$ Center for Automation and Robotics (CAR) \\ Consejo Superior de Investigaciones Científicas \\ Campo Real km 0.200, Arganda del Rey, Madrid, E-28500, Spain \\ raul.deltoro, Rodolfo.haber@car.upm-csic.es
}

\begin{abstract}
Monitoring complex electro-mechanical processes is not straightforward despite the arsenal of techniques nowadays available. This paper presents a method based on Adaptive-Network-based Fuzzy Inference System (ANFIS) to estimate eccentricity of its spinning axis. The method is experimentally tested on an ultra-precision rotating device commonly used for micro-scale turning. The developed model has three inputs, two obtained from a frequency domain analysis of a vibration signal and the third, which is the device rotation frequency. A comparative study demonstrates that an adaptive neural-fuzzy inference system model provides better error-based performance indices for detecting imbalance than a non-linear regression model. This simple, fast, and non-intrusive imbalance detection strategy is proposed to counteract eventual deterioration in the performance of ultra-high precision rotating machines due to vibrations.
\end{abstract}

Keywords: neuro-fuzzy modelling, intelligent monitoring, manufacturing processes

1. Introduction. Recently new paradigms have been developed in the field of Artificial Intelligence (AI). The widespread use of AI techniques in process monitoring and control basically improves upon the inferior performance of more conventional techniques that are only effective under theoretical design conditions [1,2]. However, this argument is not enough to develop and apply AI-based methods. Synergy between signal processing strategies and AI-based techniques should also be exploited to create effective computational methods for improved real-time applications [3,4]. With this in mind, the design of qualitatively superior control and monitoring systems may be possible to enable 
the improvement of industrial processes such as precision manufacturing.

The most representative physical variables of electro-mechanical devices in precision manufacturing processes are typically nonlinear: low signal-to-noise ratio, strong influence of environmental factors, uncertainty, and a huge volume of data generated at high frequencies. There are therefore some situations where conventional methods cannot be applied for the characterisation of physical phenomena in these devices [5]. On the other hand, the use of experimental modelling techniques, AI techniques, and advanced signal processing techniques, are useful and feasible strategies which can be combined in a study of these physical processes $[6,7]$.

Recent research on precision manufacturing in the field of nanotechnology has applied hybrid AI techniques and conventional control algorithms to reduce uncertainty in process models and to increase the accuracy of devices in ultra-precision positioning systems [8]. Moreover, these same techniques have been used to enhance the performance of actuators and systems in ultra-precision machining processes [9].

The aim of this work and its main contributions are in the development of a procedure and a model based on frequency domain analysis combined with the use of hybrid artificial intelligence techniques. The model estimates information from a high-range of signal frequencies with high levels of uncertainty in complex electro-mechanical processes. It is applied to estimate the micro-scale eccentricity of the spinning axis of a rotating device with ultra-precision performance requirements. This method can be effectively applied to reduce systemic error and decrease production time in manufacturing processes.

The paper is organized as follows: Section 2 introduces the use of ultra-precision rotating devices in manufacturing operations and presents a mathematical model of vibration in rotating devices; Section 3 describes an experimental analysis to study the relationship between vibrations and shaft eccentricity in an ultra-precision rotating device; Section 4 introduces a neuro-fuzzy model that uses frequency domain information to estimate eccentricity in a rotating device. Finally, some conclusions are drawn on the results in Section 5.

2. Ultra-precision Rotation Devices in Manufacturing Processes. In high-precision manufacturing processes, the use of rotational and linear actuators with ultra-precision requirements is necessary. A device is considered "ultra-precise" when it reaches the highest possible dimensional accuracy of state-of-the-art technology [10]. Operations such as milling, turning, drilling, etc., to produce components with micro or nano-scale features, are performed by machine tools with micro-metric resolution of their positioning axes. This precision is achieved by employing linear motors and rotational actuators with hydrostatic or magnetic bearings, and by incorporating an advanced control system with laser interferometer metrology. These advanced devices and drivers avoid stiction and reduce the influence of vibrations, friction and thermal deviation.

Despite state-of-the-art computational and mechanical technology in rotational devices, non-controlled dynamics of the positioning system continue to affect the surface quality and the dimensional accuracy of manufactured parts. Vibrations that result from these 
dynamic forces affect the desired precision. In rotational actuators, such as the air bearing spindle of ultra-precision machine tools, the dynamic forces produced during rotary movement [11] reflect these uncontrolled dynamics. The increase in dynamic forces, due to the dynamic mass imbalance of the spindle, generates an eccentricity on its rotational axis with its corresponding vibration. This vibration has a direct influence on the precision of the manufactured part.

2.1 Eccentricity and Vibrations Due to Mass Imbalance. Eccentricity in the shaft of a rotating device occurs when its centre of mass differs from its geometric centre [12]. One of the most common causes is device mass imbalance, which is produced, mainly, by the unequal distribution of mass within its components. Eccentricity in the shaft can generate dynamic forces that cause vibrations that are synchronous to the rotation frequency of the device.

Figure 1 depicts a physical representation of a rotor, rotating at speed $\omega$ and a point with mass $m$ causing an imbalance. The imbalance mass is also characterized by an eccentricity vector $e$ with its origin in the rotor axial axis and angle $\omega t$. The amplitude of the generated force $\boldsymbol{F}$ and its components, $\boldsymbol{F x}$ and $\boldsymbol{F y}$, due to the imbalance mass, [13] are calculated as:

$$
\begin{aligned}
& F x=m e \omega^{2} \cos \omega t \\
& F y=m e \omega^{2} \sin \omega t
\end{aligned} \Rightarrow F=m e \omega^{2}
$$

These forces constitute a harmonic excitation to the rotating device, causing vibrations in the same direction and frequency of the excitation force [14]. The rotating device may be considered a spring-mass-damper system, with $c_{y}$ and $k_{y}$ as its respective coefficients of viscosity and elasticity (see Figure 1), in order to construct a mathematical estimate of its vibrations. For the sake of clarity, excitation is only considered in one direction. The elasticity and viscosity of other rotor components (e.g., the bearings) are not directly considered in this analysis.

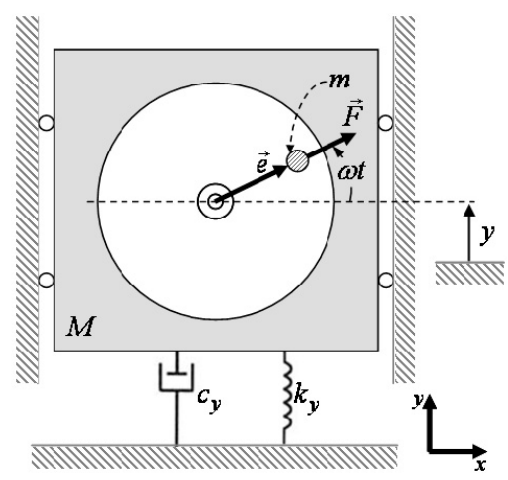

FIGURE 1. Physical model of a rotary device with imbalance mass [14]. 
If $y$ is the displacement of the non-rotational mass $(M-m)$ from the equilibrium position, the general equation of motion due to the amplitude of the imbalance force component $\boldsymbol{F y}$, can be expressed as follows:

$$
M \frac{d^{2} y}{d t^{2}}+c_{y} \frac{d y}{d t}+k_{y} y=\left(m e \omega^{2}\right) \sin \omega t
$$

The solution to Equation (2) has two parts: the homogeneous and the particular solution. The homogeneous solution describes the transient behaviour of the system, which is a free vibration that can be under damped, over damped, or critically damped [15]. At steady state, the response of the system is characterized by the particular solution of the equation, which is an oscillatory vibration of the same frequency as the excitation $F y$ with amplitude $Y$ and phase $\phi$ :

$$
y=Y \sin (\omega t-\phi) ; \quad Y=\frac{m e \omega^{2}}{\sqrt{\left(k_{y}-M \omega^{2}\right)^{2}+\left(c_{y} \omega\right)^{2}}}, \phi=\tan ^{-1} \frac{c_{y} \omega}{k_{y}-M \omega^{2}}
$$

The acceleration of motion could be expressed as:

$$
y=-\omega^{2} Y \sin (\omega t-\phi)
$$

Equations (3) and (4) represent an approximated relationship between the eccentricity, caused by the imbalance mass, and vibrations that take place in a rotating device. The amplitude of both vibration displacement and its acceleration are proportional to the imbalance mass and its eccentricity, dependent upon its rotation at speed.

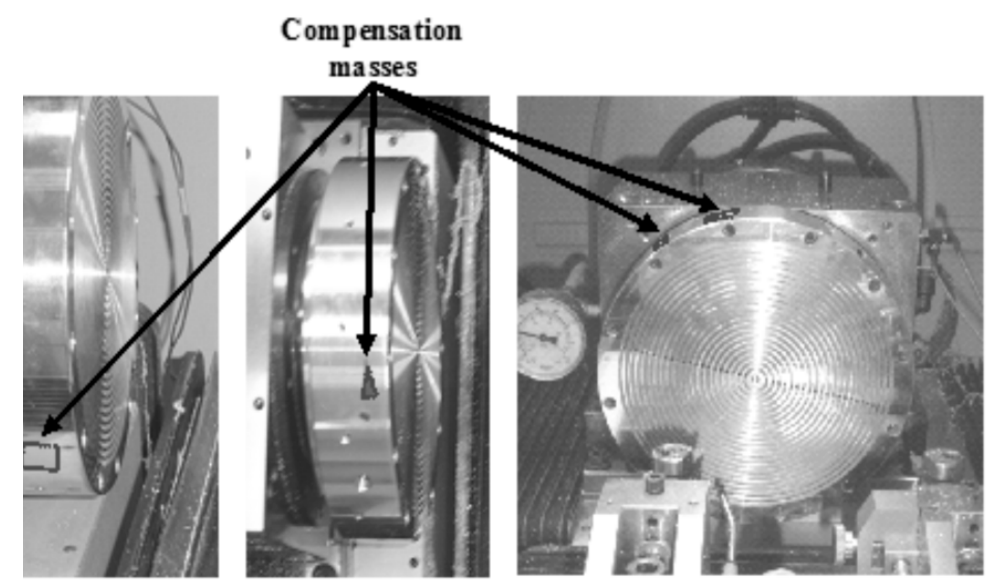

FIGURE 2. One way of compensating imbalance in high precision rotational devices. 
Modern-day methods to balance ultra-precision rotation devices are usually based on determining the position and magnitude of the imbalance masses, and then adding compensatory masses to the rotor (of the same magnitude, and diametrically opposite), as shown in Fig. 2. Some machines include manual procedures or special modules with which to carry out the compensation, in this case usually based on measuring the eccentricity of the axis at different points by means of capacitive or inductive sensors, and determining the position of greatest eccentricity. The compensation involves manually adding masses to this position until the eccentricity is within the bounds required for the corresponding process.

3. Experimental Analysis. An experimental platform was installed on a Precitech Inc. SP-150 spindle, mounted on an ultra-precision lathe, in order to conduct an experimental study of the relationship between vibration and shaft eccentricity. These types of machines are employed for finishing operations on curved and flat surfaces of both brittle and ductile materials, with very low error tolerances. Components (e.g., an optical lens) can be manufactured with arithmetic average surface roughness of below 10 nanometers and a few hundred nanometers of form accuracy.

Vibrational signals were measured with two accelerometer sensors rigidly attached to the spindle housing (see Figure 3). The sensor was a 352C15 model from PCB Piezotronics, with a sensitivity of $10 \mathrm{mV} / \mathrm{g}$ and a bandwidth of $12 \mathrm{kHz}$. The vibration signals of the $\mathrm{X}$ and $\mathrm{Y}$ spindle axes were acquired and processed with a high-performance National Instruments PXI-8187 processor, at a sample frequency of $50 \mathrm{kHz}$.
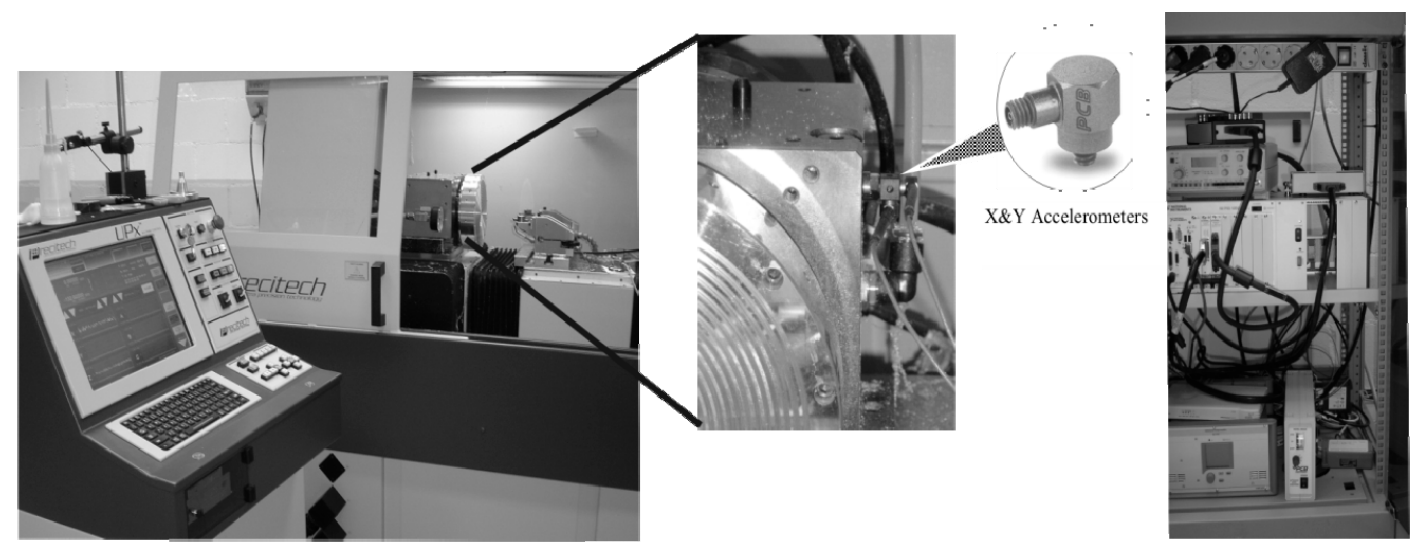

FIGURE 3. Experimental platform for imbalance detection in a high-precision device.

The ultra-precision lathe is part of a functioning production line in an industrial environment. The experimental platform does not, therefore, interfere with the manufacturing process.

The eccentricity reference value is obtained from a measurement system embedded in the Computer Numerical Control (CNC) of the lathe. The amplitude and phase of this value, displayed on the $\mathrm{CNC}$ graphical user interface, correspond to the maximum eccentricity 
position of the spindle shaft. These values may only be obtained prior to each manufacturing operation. Only the amplitude of eccentricity is used as a reference value for the experimental analysis.

Different operational conditions of the spindle have been considered, in order to analyse the relationship between vibrational level and shaft eccentricity, as shown in Table 1 . Eccentricity is manually induced by adding imbalanced masses on the spindle.

TABLE 1. Operational conditions of the experiments.

\begin{tabular}{ccc}
\hline $\begin{array}{c}\text { Spindle speed } \\
\text { [RPM] }\end{array}$ & $\begin{array}{c}\text { Eccentricity } \\
\text { [nm] }\end{array}$ & $\begin{array}{c}\text { Samples } \\
\text { for FFT }\end{array}$ \\
\hline 1000 & $13 ; 440$ & 60000 \\
3000 & $18 ; 304$ & 20000 \\
4000 & $17 ; 155$ & 15000 \\
\hline
\end{tabular}

Only the X-axis spindle vibration signal is analyzed in this study. Fast Fourier Transform (FFT) is applied to this signal, generating a frequency spectrum. A sample size proportional to the ratio between the signal sample frequency $(50 \mathrm{kHz})$ and the rotation frequency is used for each transform (see last column of Table 1), thus the frequency step in the spectrum is a fraction $(1 / 20)$ of the spindle speed.

a)

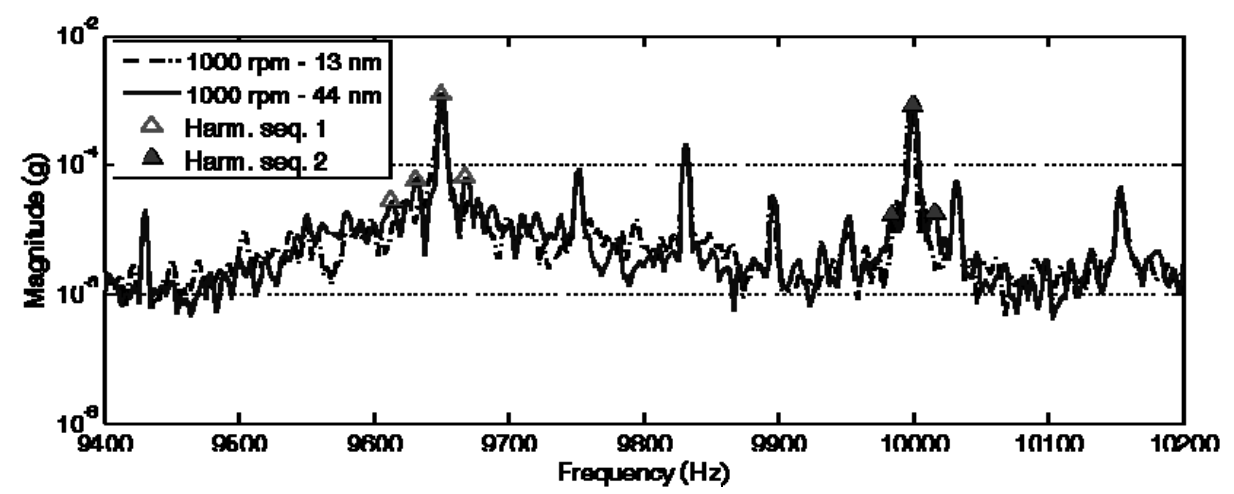

b)

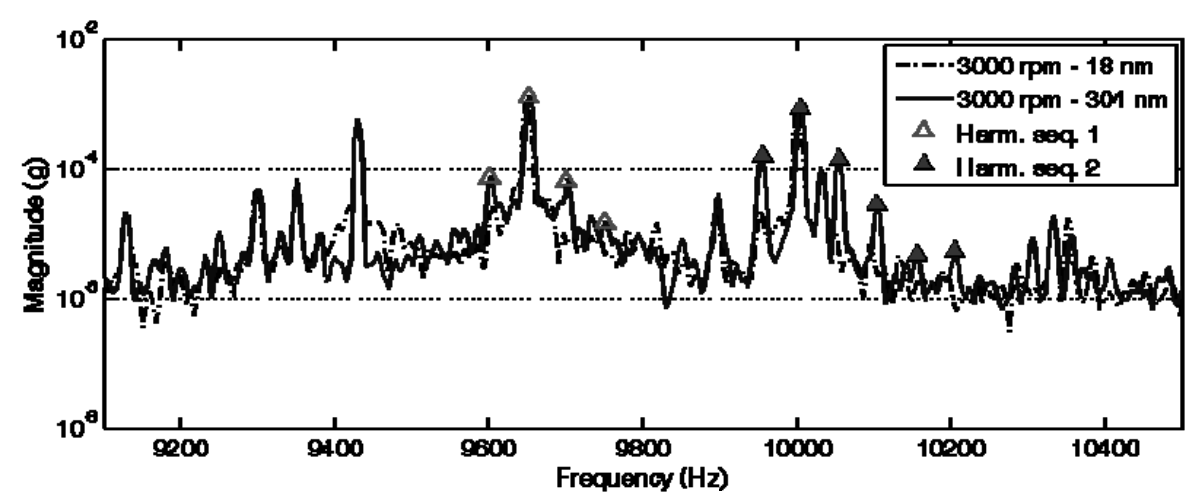


c)

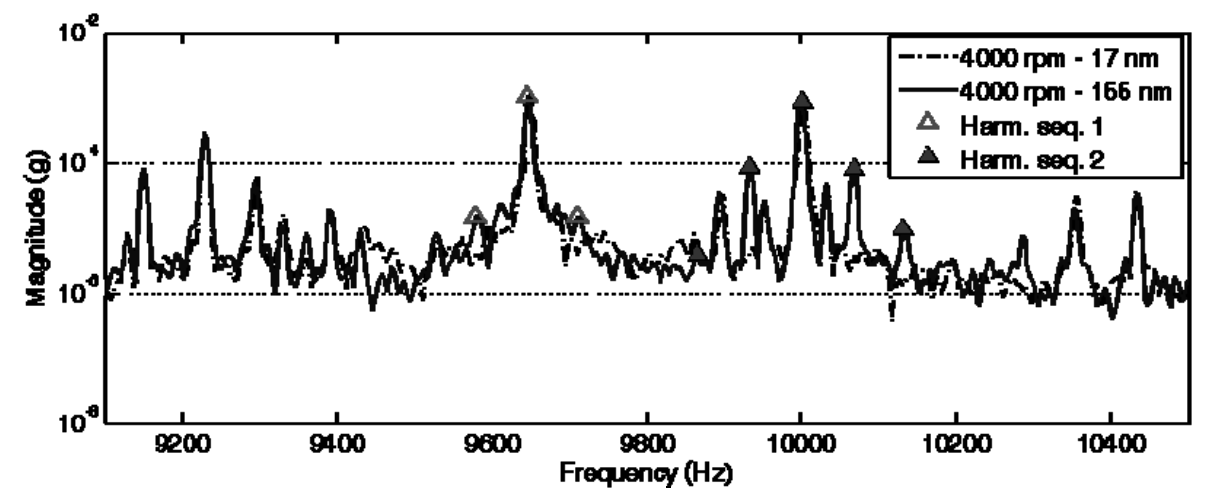

FIGURE 4. Magnitude spectra of the X-axis vibrational signal for different rotational speeds. (a) 1000 RPM, eccentricity of $13 \mathrm{~nm}$ (dash-dot line) and $44 \mathrm{~nm}$ (solid line). (b) $3000 \mathrm{RPM}$, eccentricity of $18 \mathrm{~nm}$ (dash-dot line) and $304 \mathrm{~nm}$ (solid line). (c) $4000 \mathrm{RPM}$, eccentricity of $17 \mathrm{~nm}$ (dash-dot line) and $155 \mathrm{~nm}$ (solid line).

Figure 4 shows the magnitude spectrum on a logarithmic scale for each operational condition in a frequency range close to $10 \mathrm{kHz}$, where the experiments with the same rotational frequency are depicted in the same graph. For cases with the largest eccentricity values, the sequences of the sideband harmonic frequencies appear close to those of the main harmonics of the spectrum. The harmonics within each sequence are separated by the spindle rotational frequency. It is therefore possible to estimate the eccentricity of the spindle shaft from the amplitude of the harmonic sequences.

4. Model for eccentricity estimation. One way of estimating eccentricity is to develop a model-based strategy that uses frequency-domain and time-domain information (Figure 5). The proposed model has three inputs, two obtained from a frequency domain analysis of a vibration signal $\left(A T_{1}\right.$ and $\left.A T_{2}\right)$ and the third, which is the device rotation frequency $(R F)$. The model output $\left(U L_{E}\right)$ is the maximum eccentricity in the spindle rotational axis due to the mass imbalance.

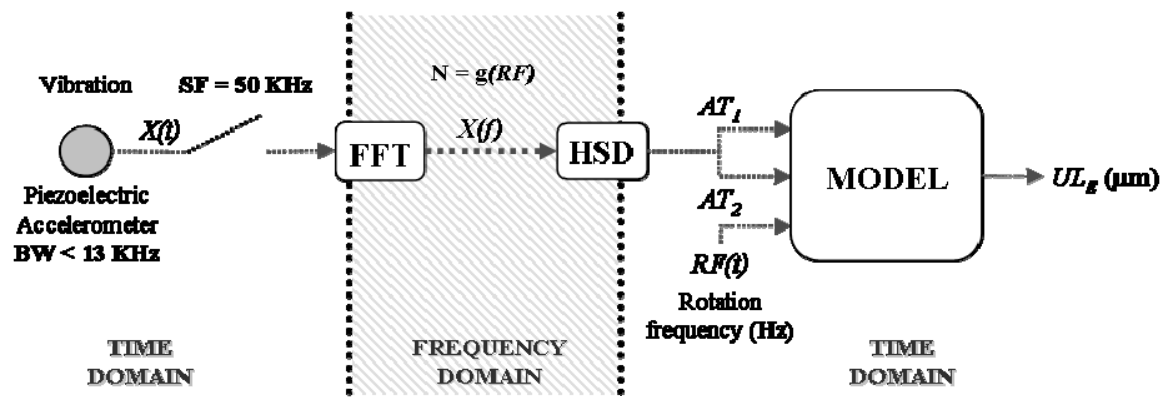

FIGURE 5. Outline of the eccentricity estimation procedure.

Figure 5 depicts an outline of the estimation procedure of eccentricity, where HSD corresponds to a spectral analysis procedure [15]. In this step, an algorithm should seek 
harmonic sidebands that form sequences around the main harmonics within a desired frequency range of the signal spectrum. The values of sample frequency (SF) and signal bandwidth (BW) correspond to those used in the experimental platform.

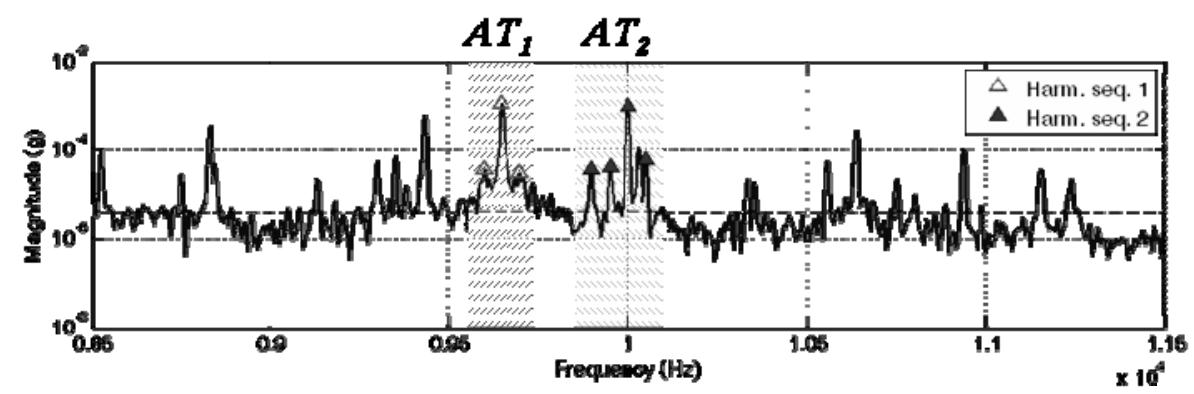

FIGURE 6. Spectral regions used for the model.

Model inputs $A T_{1}$ and $A T_{2}$ are calculated as the total sum of the amplitudes of the sideband harmonics identified around the two principal harmonics. Thus, the value of each $A T_{i}$ input is only related to the harmonics that are generated due to eccentricity.

$$
A T_{i}=\sum_{j=1}^{n} A_{j, i}
$$

$A_{j, i}$ represents the amplitude of each $j$ sideband harmonic around the $i$-th principal harmonic, where $n$ is the total of new harmonics within a sequence.

Figure 6 illustrates the spectral regions from which inputs $A T_{1}$ and $A T_{2}$ would be taken. These regions are not fixed and are determined by the principal harmonic frequencies, and their width depends on the frequencies of the sideband harmonics.

\subsection{Regression Model for Eccentricity Estimation}

A direct model represented by a hyperbolic tangent function with a correlation coefficient (R2) equal to 0.98 was obtained by fitting an experimental data set through regression techniques and the harmonic detection algorithm. The single-input single-output model relates the eccentricity $\left(U L_{E}\right)$ of the spindle shaft with the power ratio $(P R A)$ of the detected sequence around the spectrum's principal harmonic. The model for estimating the eccentricity is obtained by inversion.

$$
\begin{gathered}
P R A=\frac{1}{2}\left[\tanh \left(m \cdot U L_{E}+n\right)+1\right] \\
U L_{E}=\left\{\begin{array}{l}
U L_{E m i n}, \quad P R A \leq P R A_{\text {min }} \\
\frac{1}{m}\left[\frac{1}{2} \ln \left(\frac{P R A}{1-P R A}\right)-n\right], P R A>P R A_{\text {min }}
\end{array}\right.
\end{gathered}
$$




$$
P R A_{\min }=\frac{1}{2}\left[\tanh \left(m \cdot U L_{E \min }+n\right)+1\right]
$$

In the above equations, the linear fit coefficients $m$ and $n$ are initially set at 2.220126 and -1.59696 , respectively. The parameters ULEmin and PRAmin are defined as minimum specifications of the eccentricity and the power ratio, respectively. The minimum value ULEmin was set to $0.010 \mu \mathrm{m}$, in accordance with the range of the experimental dataset. These parameters are introduced to take into account the sensitivity of the piezoelectric sensor and the vibration attenuation due to the spindle's mechanical properties.

4.2 Takagi-Sugeno Type Neuro-fuzzy Inference System for Eccentricity Estimation. The Takagi-Sugeno type fuzzy inference system represents an excellent alternative technique for designing a model that ensures accurate eccentricity estimation, due to its capabilities such as interpolation and function approximation. The use of AI hybridization techniques such as artificial neural networks and fuzzy inference systems can facilitate the model design procedure.

Among the new hybrid systems, the Adaptive Network based Fuzzy Inference System (ANFIS), as well as pioneering work, is also the simplest approach in terms of computational time, which makes it feasible for real-time applications [16-18]. Its principle is based on the extraction of fuzzy rules at each level of a neural network. Once the rules have been obtained, they provide the necessary information on the overall behaviour of the modelled system.

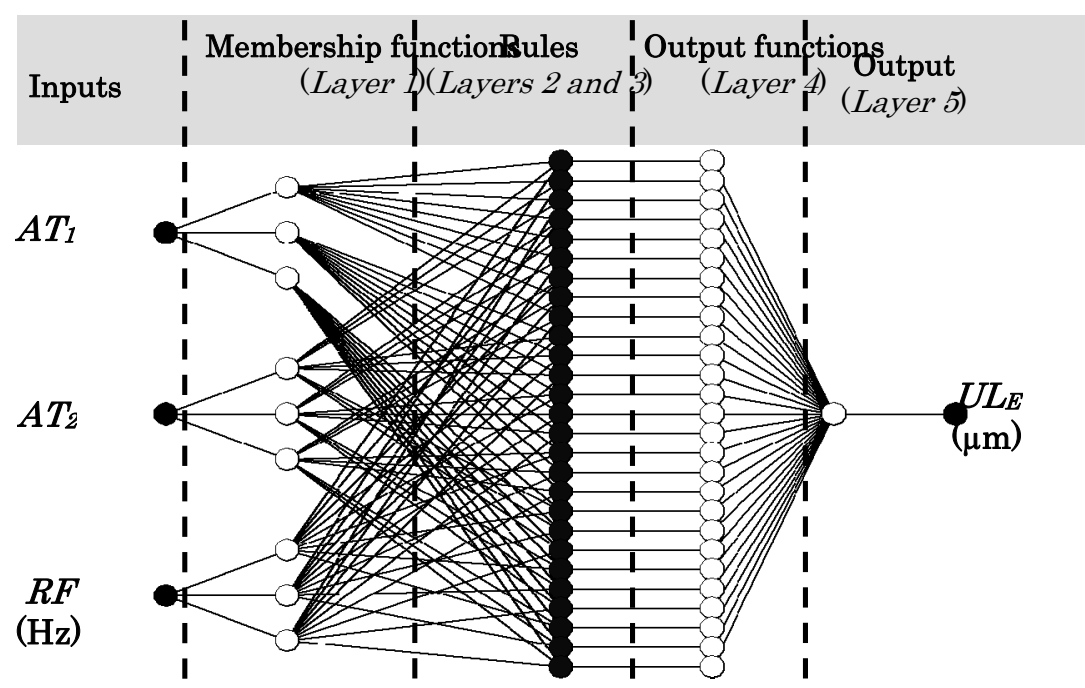

FIGURE 7. ANFIS model structure.

ANFIS implements the Takagi-Sugeno model for the structure of the fuzzy system's if-then rules. The ANFIS architecture has five layers: the premise parameters layer (1), calculation of the firing strength of each rule (2), a layer to normalize the firing strength of 
each rule (3), the consequent parameters computation layer (4), and a final layer (5) to calculate the overall output as the summation of the outputs from the previous layer. Figure 6 shows the proposed model with its corresponding ANFIS layers.

$$
f(x)=\frac{1}{1+\left(\frac{x-p}{m}\right)^{2 n}}
$$

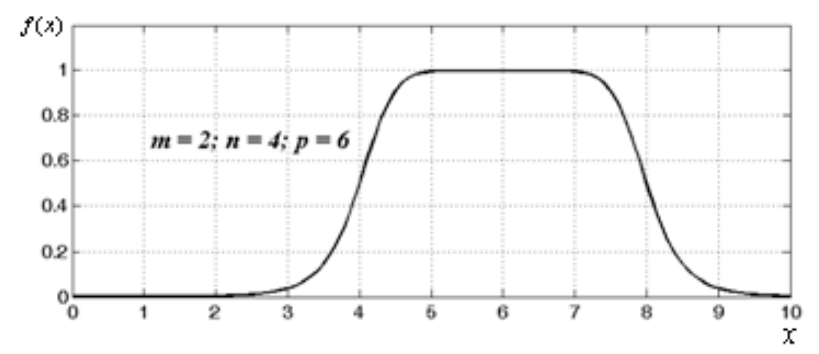

FIGURE 8. The generalized bell distribution function and a graphic example.

Each model input is divided into three partitions, using an extension of the Cauchy-Lorentz distribution, also called a generalized bell function, as the membership function. A typical example of this function is shown in Figure 8. The output of each rule is a linear combination of the inputs, and the model output is the sum of all the rules. The inputs $A T_{1}$ and $A T_{2}$ have magnitudes of vibration (acceleration) from the English system of units, in this case milli-g $\left(1 \mathrm{~g} \approx 9.8 \mathrm{~m} / \mathrm{s}^{2}\right)$. The values that take the rotation frequency input $(R F)$ are expressed in Hertz $(\mathrm{Hz})$ and the model output is estimated in micrometers $(\mu \mathrm{m})$.

The model is trained using a hybrid mode, where the antecedent of the rules (membership functions) is determined employing error back propagation as the learning strategy and the consequent of each rule is estimated using the least squares method (output functions). In the first step or 'forward pass', the input models are propagated, and the optimum consequent fuzzy sets are estimated with an iterative least squares procedure, while the premises remain fixed. In the second step or 'backward pass', the back propagation procedure is used to modify the premise parameters, and the consequents remain constant. The training process stops whenever the designated epoch number (iteration count) is reached or the training error goal is achieved. The results of the training are shown in Figure 9.

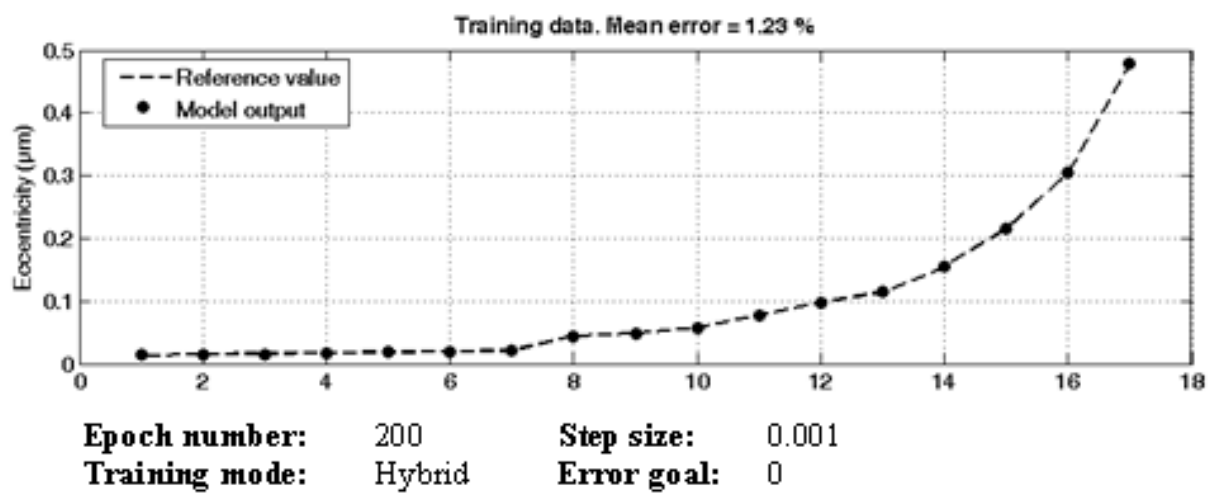

FIGURE 9. Training results and training data. 
The universes of discourse of the variables were taken to be $A T_{1} \in[0,50] \quad, \quad A T_{2} \in[0,142](\mathrm{mg}) \quad, \quad R F \in[16.67,83.33](\mathrm{rev} / \mathrm{s})$, and $U L_{E} \in[0.013,0.478](\mu m)$.

The output of each rule is a linear combination of the three inputs, and the model output is the sum of all the rules:

$$
U L_{E}^{R i}=a_{i} \cdot A T_{1}+b_{i} \cdot A T_{2}+c_{i} \cdot R F+d_{i}
$$

where, $a_{i}, b_{i}, c_{i}, d_{i}$ are the coefficients obtained by the training corresponding to the $i$-th rule, and $U L_{E}^{R i}$ is the output of the $i$-th rule.

The input membership functions are shown in Fig. 10. The rules of the knowledge base are automatically created from the possible combinations of the logistic variables of each model input. The antecedent of each rule is related through the connective "AND", and its consequent is calculated from a linear affine function.
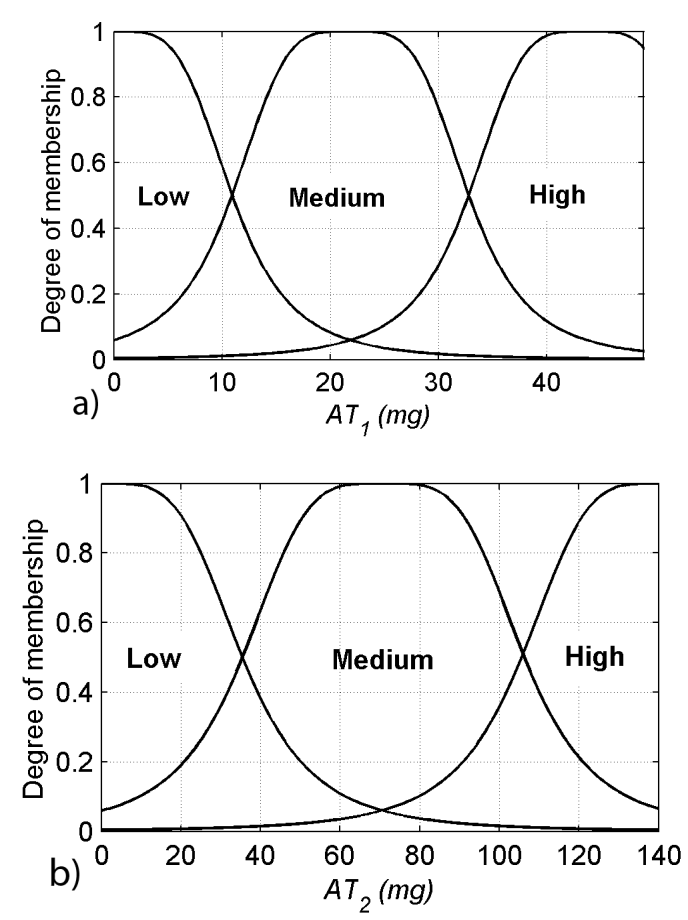


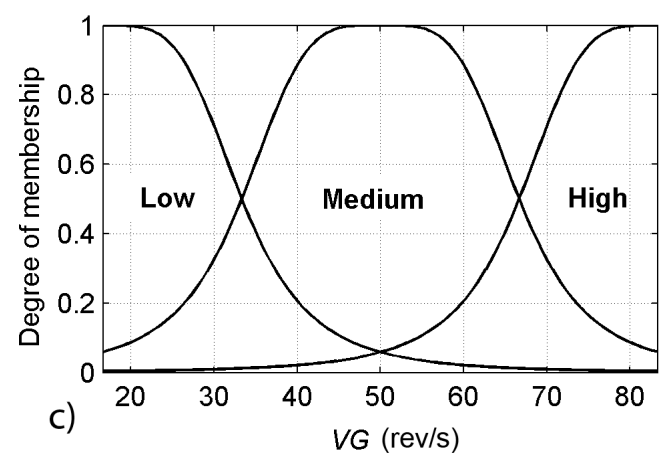

FIGURE 10. Membership functions of the inputs of the ANFIS model.

The rules have been defined as follows:

R1: IF ( $A T_{1}$ is Low) $A N D$ ( $A T_{2}$ is Low) AND (RF is Low) THEN (UL $L_{M}$ is $\left.U L_{R I}\right)$ (1)

R2: IF ( $A T_{1}$ is Low) $\boldsymbol{A N D}$ ( $A T_{2}$ is Low) $\boldsymbol{A N D}$ (RF is Medium) THEN (UL $L_{M}$ is $\left.U L_{R 2}\right)$ (1)

R3: IF ( $A T_{1}$ is Low) $A N D$ ( $A T_{2}$ is Low) $A N D$ ( $R F$ is High) THEN ( $U L_{M}$ is $U L_{R 3}$ ) (1)

R27: IF ( $A T_{1}$ is High) AND ( $A T_{2}$ is $\left.\mathrm{High}\right)$ AND (RF is High) THEN ( $U L_{M}$ is $\left.U L_{R 27}\right)(1)$

4.3 Model Validation. This section explains how the model is validated with another data set obtained experimentally. Experiments were performed with spindle speeds of 1000 , $2000,3000,4000$ and $5000 \mathrm{r} / \mathrm{min}(16.667-83.333 \mathrm{~Hz})$ and shaft eccentricity values from 13 to $478 \mathrm{~nm}$ were introduced by manually adding imbalanced masses to the spindle.

TABLE 2. Data for validation and error analysis of the ANFIS model.

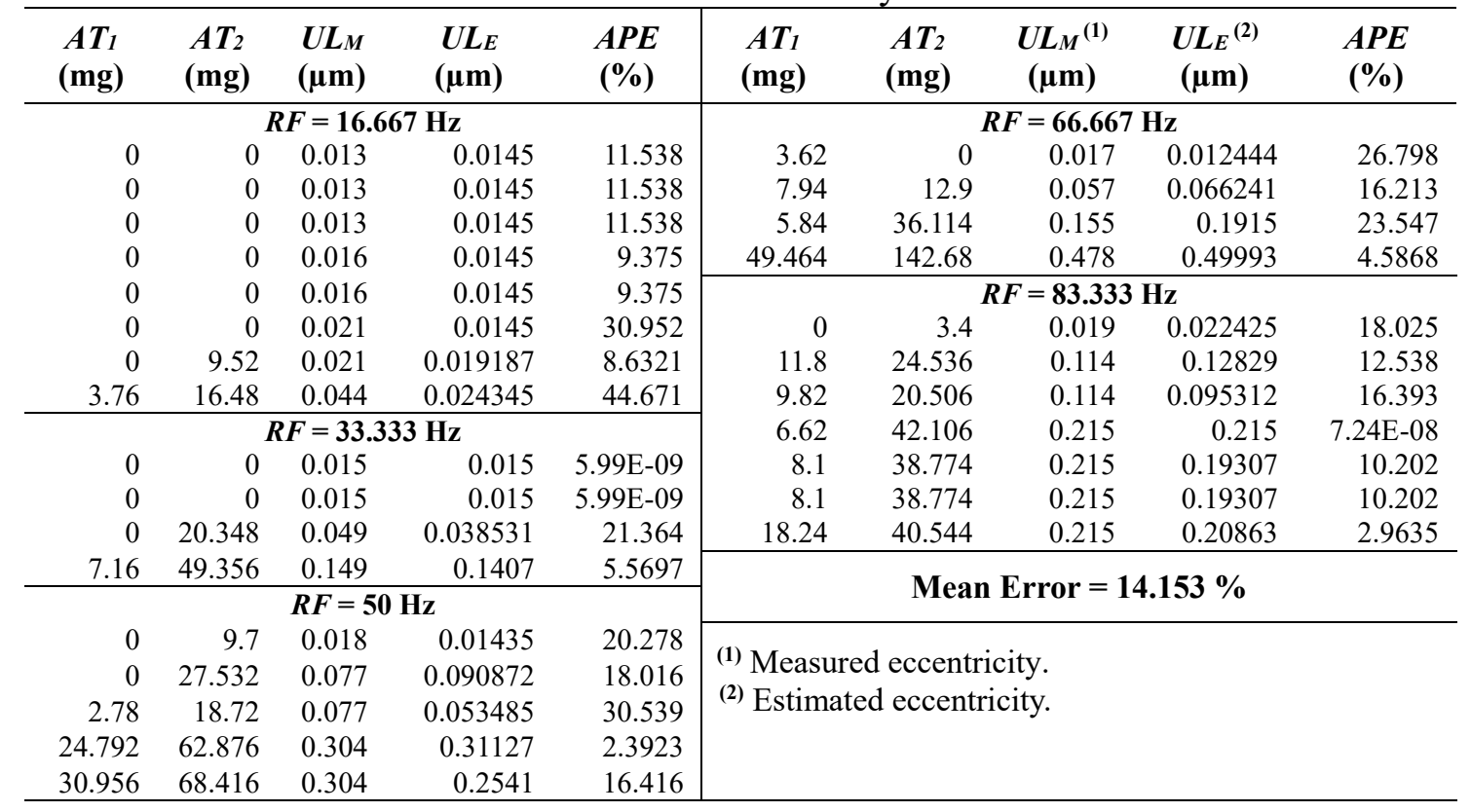


Table 2 shows the experimental data used for each input-output model. The columns headed $\boldsymbol{U} \boldsymbol{L}_{\boldsymbol{M}}$ and $\boldsymbol{U} \boldsymbol{L}_{\boldsymbol{E}}$ correspond to the output reference value (measured eccentricity) and the model eccentricity estimation, respectively. The model's inputs $\left(\boldsymbol{A} \boldsymbol{T}_{1}\right.$ and $\left.\boldsymbol{A} \boldsymbol{T}_{2}\right)$ were calculated from the spectral analysis, within a range of around $10 \mathrm{kHz} \pm 15 \%$, of the vibration signal of the spindle $\mathrm{X}$-axis.

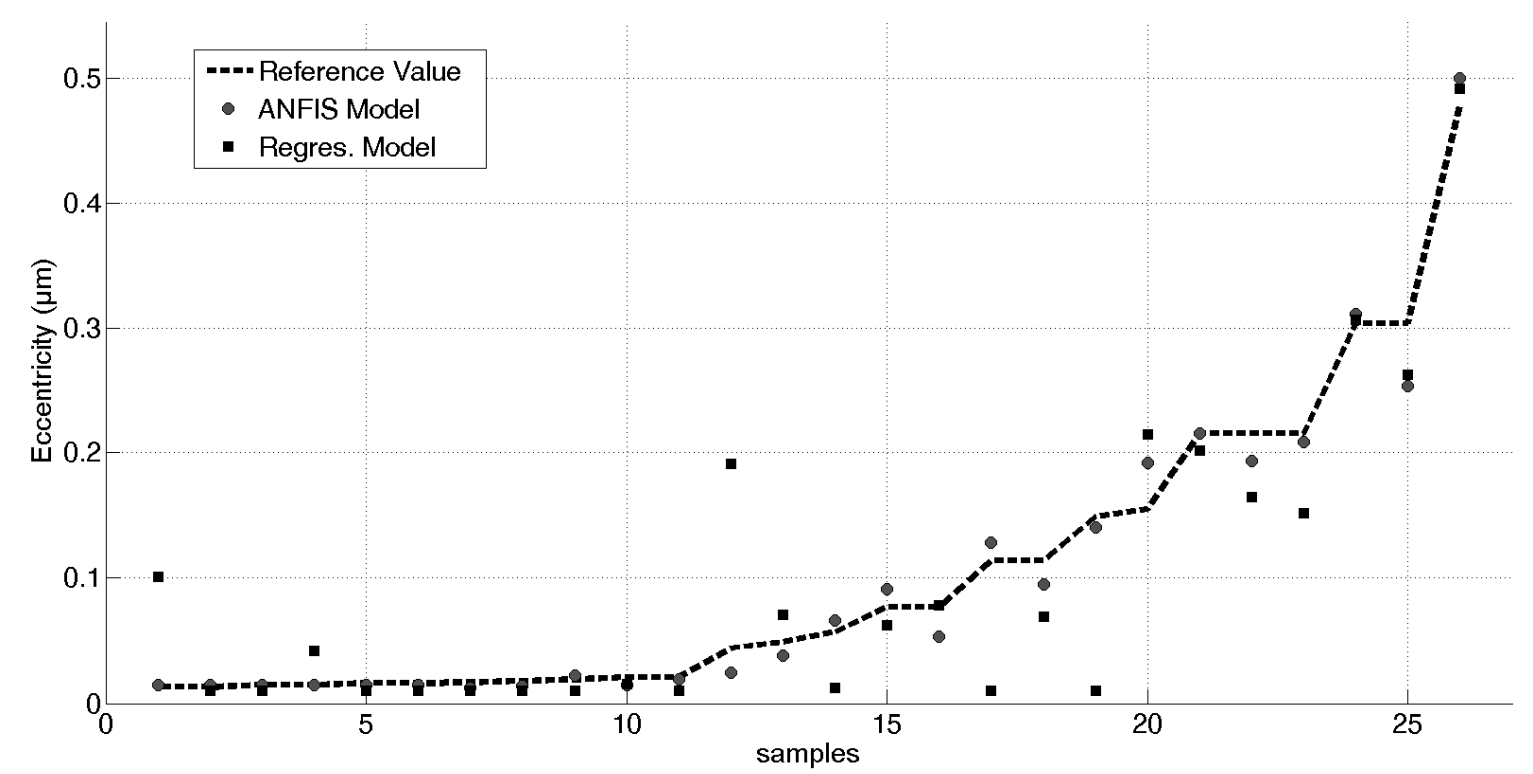

FIGURE 11. ANFIS model validation. Estimated eccentricity and reference value.

A comparative study was conducted with a regression model, in order to evaluate the behaviour of the ANFIS model. Five performance indices were considered in the experimental study - the sum of squared errors SSE, the sum of average errors SAE, the mean square error MSE, the mean absolute error MAE, and the maximum error. The results are shown in Table 3.

TABLE 3. Comparison of the two modeling techniques considered in this study.

\begin{tabular}{cccccc}
\hline $\begin{array}{c}\text { Algorithm/Error } \\
\text { Criterion (\%) }\end{array}$ & $\begin{array}{c}\text { SSE } \\
\left(10^{4}\right)\end{array}$ & SAE & MSE & MAE & $\begin{array}{c}\text { Max } \\
\text { Error }\end{array}$ \\
\hline ANFIS & 0.83 & 371.92 & 17.89 & 14.15 & 44.67 \\
\hline Regression & 64.3 & 2013.6 & 157.25 & 77.44 & 93.28 \\
\hline
\end{tabular}

Figure 11 shows the behaviour of the two models considered in this study in relation to the eccentricity measured on the experimental platform described in Section 2. It is evident that ANFIS outperformed the regression model. Figure 11 depicts the validation data, where the average error between the model output and the desired output is $14.15 \%$, which shows good 
performance of the model within the full range of analysis.

The absolute percentage error for validation data is illustrated in Figure 12a, from which it is clear that the model has quite high uncertainty at the lowest eccentricity estimation interval. This decreases as the eccentricity estimation value increases, but in general, the model error is below 20\% throughout the range under analysis. Furthermore, this range is divided into three intervals, selected and classified according to the degree of accuracy that they each represent and the estimation uncertainty. The eccentricity values of between $0.012-0.030 \mu \mathrm{m}$ represent the highest accuracy and the highest uncertainty prediction. The second region between $0.030-0.150 \mu \mathrm{m}$ represents high accuracy and high uncertainty. The third region between $0.150-0.500 \mu \mathrm{m}$ represents medium accuracy and low uncertainty. The average error is calculated for each region (see Figure 12b).

a)
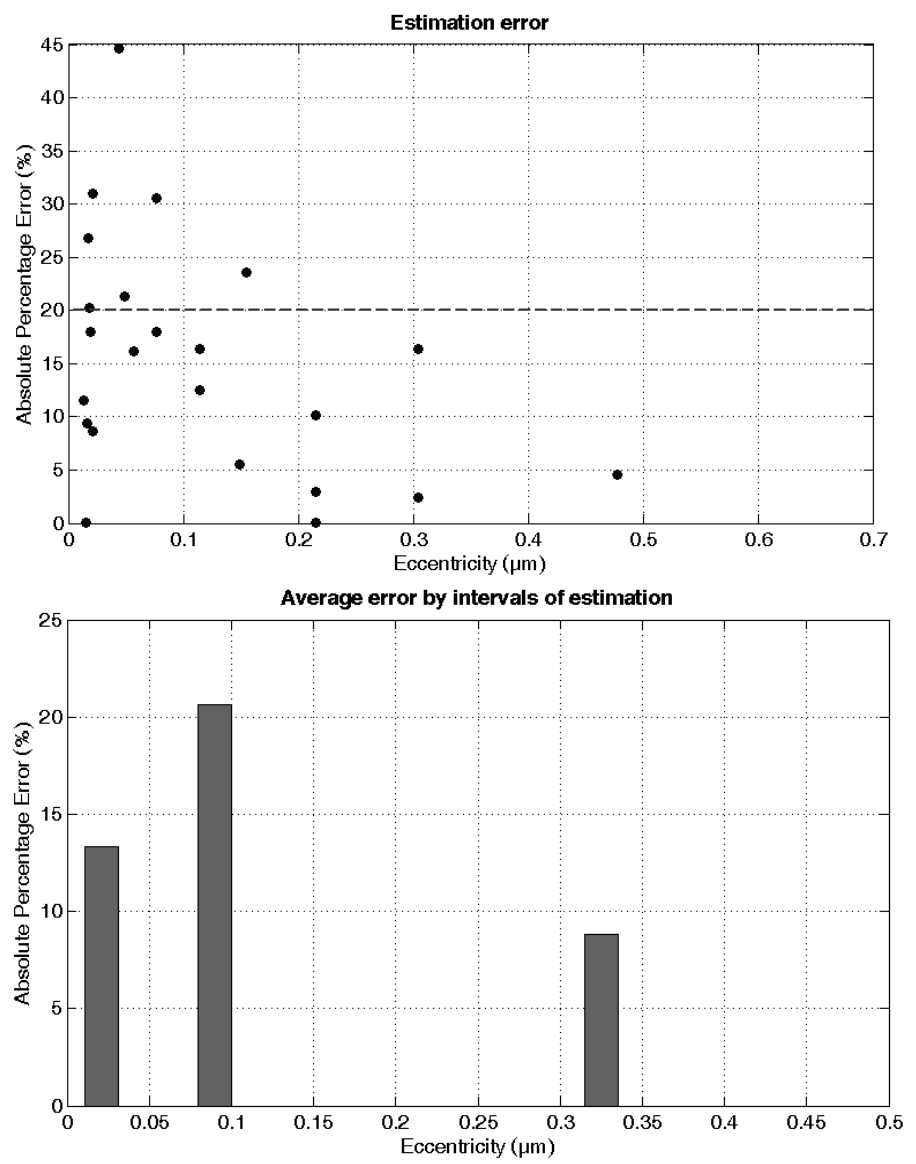

FIGURE 12. Model estimation errors: a) absolute percentage error vs. the eccentricity reference value, $\mathbf{b}$ ) average error by intervals of estimation.

The model error is less than $15 \%$ but increases in the middle interval as shown in Figure 10b. This performance is more evident in the regions of highest and medium accuracy. Indeed, the model's performance is adequate according to the required scale of the estimated variable and from the standpoint of industrial precision in manufacturing. The 
presence of high uncertainty in some regions of estimation is due to low vibration magnitudes. The stiffness of the rotation device and the sensitivity of the measurement system (e.g.: accelerometer sensor sensitivity) are the main causes of low-level vibrations. These areas of lower mathematical certainty correspond to lower regions of training input in the mechanical and measuring devices.

5. Conclusions. A procedure to estimate eccentricity in ultra-precision rotating devices has been discussed in this work. It is based on a model that implements an Adaptive-Network-based Fuzzy Inference System (ANFIS). The three model inputs are information from the frequency and time domains. The technique has been applied to an ultra-precision rotating device to estimate eccentricity at its spinning axis, caused by its inertial mass imbalance. Its eccentricity is estimated during the rotary movement of the device from its steady state vibrations, and the developed models are adjusted and validated with experimental data. Furthermore, a comparison with a regression model demonstrated the potential of an adaptive neural-fuzzy inference system which provided better accuracy than a regression model. In brief, the proposed strategy for imbalance detection in ultra-high precision rotating machines is simple, fast, and non-intrusive in a production environment.

Finally, the proposed model has performed to an acceptable level which demonstrates that experimental modelling techniques combined with neurofuzzy and signal processing techniques can be satisfactorily applied to real-time intelligent monitoring of devices with micro-scale precision requirements.

\section{REFERENCES}

1. Haber, R.; Alique, J. R.; Ros, S.; Peres, C. R., Fuzzy Supervisory Control of End Milling Process. Information Sciences 1996, 89, (1-2), 95-106.

2. Haber, R. E.; Haber, R.; Alique, A.; Ros, S., Application of knowledge-based systems for supervision and control of machining processes. Handbook of software engineering and knowledge engineering 2002, 2, 673-710.

3. Beruvides-López, G.; Quiza-Sardiñas, R.; Haber-Guerra, R.; Del Toro-Matamoros, R., Features extraction from signals for indirect tool condition monitoring in microdrilling. Dyna (Spain) 2013, 88, (4), 405-413.

4. Penedo, F.; Haber, R. E.; Gajate, A.; Del Toro, R. M., Hybrid incremental modeling based on least squares and fuzzy K-NN for monitoring tool wear in turning processes. IEEE Transactions on Industrial Informatics 2012, 8, (4), 811-818.

5. del Toro, R. M.; Schmittdiel, M. C.; Haber-Guerra, R. E.; Haber-Haber, R. In System identification of the high performance drilling process for network-based control, ASME 2007 International Design Engineering Technical Conferences and Computers and Information in Engineering Conference, 2007; American Society of Mechanical Engineers: 2007; pp 827-834.

6. Haber, R. E.; Cantillo, K.; Jiménez, J. E., Networked sensing for high-speed machining processes based on CORBA. Sensors and Actuators, A: Physical 2005, 
$119,(2), 418-426$.

7. Haber, R.; Peres, C. R.; Alique, J. R.; Ros, S. In Fuzzy supervisory control of end milling process, Proceedings of the Joint Conference on Information Sciences, 1994; 1994; pp 212-215.

8. Ho, W., Takagi-Sugeno fuzzy model of nonlinear HIV dynamics: Chebyshev-series approach integrated with genetic algorithm. International Journal of Innovative Computing, Information and Control 2012, 8, (2), 1438-1451.

9. Goto, S.; Tsukamoto, K., On-line residual life prediction including outlier elimination for condition based maintenance. International Journal of Innovative Computing, Information and Control 2012, 8, (3B), 2193-2202.

10. Altug, S.; Chow, M. Y.; Joel Trussell, H., Fuzzy inference systems implemented on neural architectures for motor fault detection and diagnosis. IEEE Transactions on Industrial Electronics 1999, 46, (6), 1069-1079.

11. Shao, Y.-E.; Lu, C.-J.; Chiu, C.-C., A fault detection system for an autocorrelated process using SPC/EPC/ANN and SPC/EPC/SVM schemes International Journal of Innovative Computing, Information and Control 2011, 7, (10), 5417-5428.

12. Taniguchi, N., Current Status in, and Future Trends of, Ultraprecision Machining and Ultrafine Materials Processing. CIRP Annals - Manufacturing Technology 1983, 32, (2), 573-582.

13. Ulmer Jr, B. C.; Kurfess, T. R., Integration of an open architecture controller with a diamond turning machine. Mechatronics 1999, 9, (4), 349-361.

14. Dukkipati, R. V., Mechanism and Machine Theory. 2nd ed.; Bohem Press: 2007; p 624.

15. Thomson, W., Theory of Vibration With Applications 4th ed.; Taylor \& Francis: 1996; p 560.

16. Jang, J.-S. R., ANFIS: adaptive-network-based fuzzy inference system. IEEE Transactions on Systems, Man and Cybernetics 1993, 23, (3), 665-685.

17. Martin, A. G.; Guerra, R. E. H., Internal model control based on a neurofuzzy system for network applications. a case study on the high-performance drilling process. IEEE Transactions on Automation Science and Engineering 2009, 6, (2), 367-372.

18. Gajate, A.; Bustillo, A.; Haber, R. E., Transductive neurofuzzy-based torque control of a milling process: Results of a case study. International Journal of Innovative Computing, Information and Control 2012, 8, (5 B), 3495-3510. 